\title{
ADAPTIVE METHODS TO SOLVE FREE BOUNDARY PROBLEMS OF FLOW THROUGH POROUS MEDIA
}

\author{
KYOON YANG CHUNG AND NOBORU KIKUCHI \\ College of Engineering, The University of Michigan, Ann Arbor, MI 48109-2125 Michigan, U.S.A.
}

\section{SUMMARY}

Grid adaptive methods combined with domain adaptation are discussed for two-dimensional seepage flow problems with free boundaries through porous media. Examples of grid and domain adaptive methods are presented to demonstrate several ways to predict grids and shapes of free boundaries using an iterative scheme. Finally, the combined adaptive methods are applied to obtain smooth non-oscillatory shape of a free boundary of seepage flow through non-homogeneous porous media.

\section{INTRODUCTION}

Although several fixed domain methods have been introduced to solve free boundary problems of flow through porous media, it is still popular to apply domain adaptive methods which adjust the flow domain and the free boundary at each iteration so that the free boundary condition can be satisfied at the last moment of the convergent iterative procedure. Among such adaptive methods to solve free boundary problems, the works by Taylor and Brown, ${ }^{1}$ Finn ${ }^{2}$ and Neuman and Witherspoon ${ }^{3}$ are fundamental. Especially, Neuman and Witherspoon provided a sophisticated two-step iterative procedure which could solve many difficult free boundary problems. After their work, major contributions in this area were introduction of various fixed domain methods which need not define new finite element grids during iteration to adjust the flow domain and the free boundary. The flow domain itself was a part of the solution to be obtained. Fixed domain methods introduced so far can be classified into two groups; the extended pressure head method and the variational inequality method. The first method is based on the concept that the pressure head $P$, which is related to the head $\phi$ and the height $y$ of a point from the datum line by $P=\gamma(\phi-y)$, where $\gamma$ is specific weight of fluid, is extended to the unsaturated area 'smoothly' from the flow domain $\Omega$. This extension of the pressure head to the entire porous media yields the extended coefficient of permeability of the medium:

$$
K_{\mathrm{ext}}=K+\frac{1}{\varepsilon}\left(1-\chi_{\Omega}\right)
$$

where $\chi_{\Omega}$ is the characteristic function of the flow domain $\Omega$ defined by

$$
\chi_{\Omega}=1 \text { if } x \in \Omega, \quad \chi_{\Omega}=0 \text { if } x \notin \Omega
$$

and $\varepsilon$ is a regularity parameter which is expected to be a very small positive real number, for example, $\varepsilon=\left(10^{-3}-10^{-5}\right) K_{\min }$ and $K_{\min }$ is the minimum of the $K$ in the flow domain. Mathematically, this extended pressure head method was introduced by Alt $^{\mathbf{4 . 5}}$ and was also studied by Brezis et al. ${ }^{6}$ Application of these mathematicians' work to solve free boundary 
problems of flow through porous media was done by Le Tallec ${ }^{7}$ and Oden and Kikuchi. ${ }^{8}$ In an engineering context this method was introduced by Desai ${ }^{9,10}$ independently of the above mathematicians' studies; he solved a number of practical problems together with experimental verification of his residual flow procedure.

The variational inequality method was first introduced by Baiocchi ${ }^{11}$ and was applied to solve free boundary problems by Baiocchi et al. ${ }^{12}$ Some extension of their works and extensive study on numerical methods were performed by Bruch ${ }^{13}$ and Oden and Kikuchi. ${ }^{8}$ This method, however, suffers a serious restriction; although it possesses a beautiful mathematical structure for its theory, application of the method to general irregular domains is almost impossible. More precisely, the method requires a kind of regular domain such as a rectangular domain.

In the present paper we shall return to domain adaptive methods, and shall provide new insight to these more or less classical methods by applying studies of grid adaptive finite element methods based on mathematical error analysis. Grid adaptive methods are regarded as schemes to improve the quality of finite element approximations by introducing refinement of grids in the area where the approximation error is large. That is, we shall consider a combination of grid and domain adaptive methods to solve free boundary problems of flow through porous media.

\section{FLOW THROUGH POROUS MEDIA WITH FREE BOUNDARIES}

A standard description of finite element methods is given in this section for a two-dimensional irrotational incompressible seepage flow through a porous dam with free boundaries. Let a flow field be denoted by $\Omega$, and let it be Lipschitz as shown in Figure 1. The boundary $\Gamma$ of the flow domain $\Omega$ consists of three mutually disjoint segments $\Gamma_{0}, \Gamma_{1}$ and $\Gamma_{2}$. The free boundary $\Gamma_{0}$ is $a$ priori unknown and satisfies the free boundary conditions. On $\Gamma_{1}$ the head is prescribed, while the flux is given on $\Gamma_{2}$.

The boundary value problem is formulated as

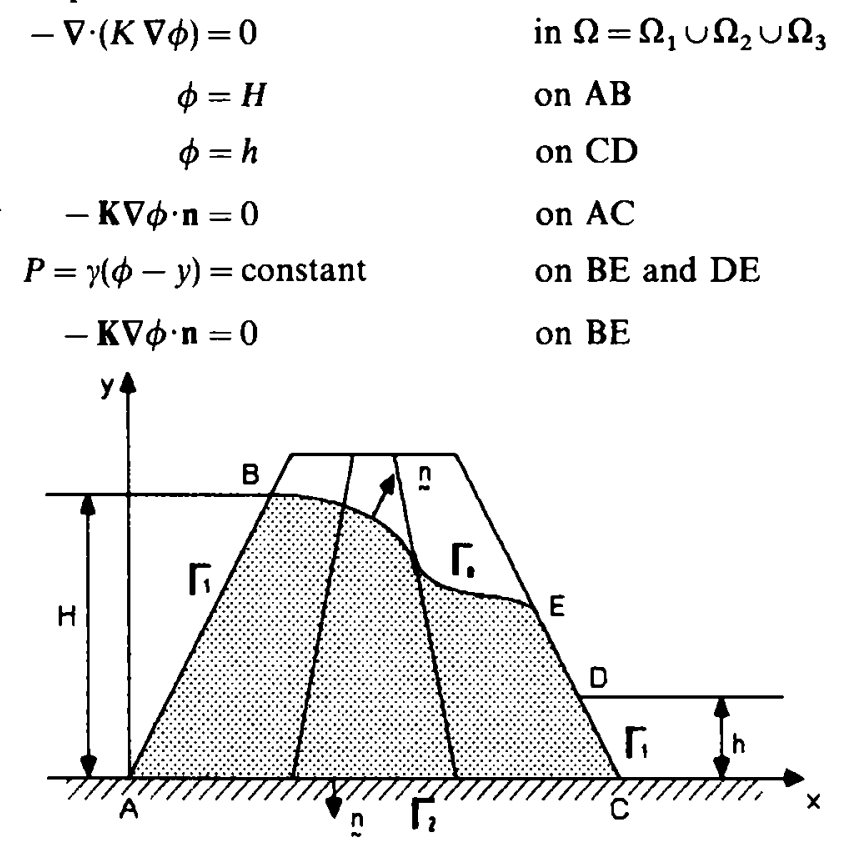

Figure 1. Example of non-homogeneous dam 
Here, $\Gamma_{1}=\mathrm{AB} \cup \mathrm{CD}, \Gamma_{2}=\mathrm{AC}, \Gamma_{0}=\mathrm{BE}, H$ and $h$ are prescribed heads, $\gamma$ is the specific weight of water, and $\mathbf{K}$ is permeability matrix of dam material. Equation (5) is referred from Darcy's law as the no-flux condition across the boundary $\Gamma_{2}$, that is, $\Gamma_{2}$ is an impermeable boundary. For simplicity, by taking zero pressure $P$, hydraulic head $\phi$ is equal to the vertical height $y$ along the free boundary $\Gamma_{0}$ and seepage surface DE. The free boundary conditions (6) and (7) must be satisfied on the free boundary whose position is also unknown $a$ priori as well as the head $\phi$.

The weak form of the local description (2) is obtained by multiplying both sides of (2) by an arbitrary $C^{0}$ continuous function $\phi$ and integrating over the domain $\Omega$, applying the divergence theorem, and substitution of the boundary condition (5) and $\bar{\phi}=0$ on $\Gamma_{1}$ :

$$
\int_{\Omega}(\nabla \phi)^{\mathrm{r}} \mathbf{K} \nabla \phi \mathrm{d} \Omega=0 \quad \text { for all } \phi \text { such that } \phi=0 \text { on } \mathrm{AB}, \mathrm{CD} \text { and } \mathrm{DE}
$$

with boundary conditions

$$
\begin{array}{ll}
\phi=H & \text { on AB } \\
\phi=h & \text { on CD }
\end{array}
$$

and the remaining free boundary conditions

$$
\begin{aligned}
& \phi=y \quad \text { on BE and DE, } \\
& \mathbf{K} \boldsymbol{\nabla} \cdot \mathbf{n}=\mathbf{0} \quad \text { on } \mathrm{BE}
\end{aligned}
$$

while (5) has been applied to obtain the form (8). It is noted that the weak form (8) is nonlinear since the flow domain $\Omega$ is unknown, i.e. the position of the free boundary $\Gamma_{0}$ and the seepage point $E$ are also unknown, although (8) is apparently linear in $\phi$. Now finite element discretization can proceed using the shape functions of four-node quadrilateral isoparametric element:

$$
\begin{aligned}
& \phi=\sum_{i} \phi_{i} N_{i}=\mathbf{N}^{\mathrm{T}} \phi \\
& \phi=\sum_{i} \phi_{i} N_{i} \quad i=1, \ldots, n
\end{aligned}
$$

where $n$ is the total number of nodes. Thus, we have a typical statement giving

$$
\begin{aligned}
& \sum_{e=1}^{E} \int_{\Omega_{e}}\left(\nabla N_{i}\right)^{\mathrm{T}} \mathbf{K} \nabla N_{j} \mathrm{~d} \Omega \phi_{j}=0 \quad i=1, \ldots, n \\
& \text { in } \Omega \text { except on } \mathrm{AB}, \mathrm{CD} \text { and } \mathrm{DE}, \text { where } \bar{\phi}=0
\end{aligned}
$$

for which $\phi=H$ on $\mathrm{AB}$ and $\phi=h$ on CD are prescribed. Here $\Omega_{e}$ denotes a discretized element and $E$ is the total number of elements. The derivatives of shape functions with respect to the global coordinates is obtained from the isoparametric relation between an element $\Omega_{e}$ in the global coordinate system $\left(x_{1}, x_{2}\right)$ and the square master element in the normalized co-ordinate system $\left(\xi_{1}, \xi_{2}\right)$. That is,

$$
\frac{\partial N_{i}}{\partial x_{j}}=\sum_{q=1}^{2}\left[J_{j q}^{-1} \frac{\partial \hat{N}_{i}}{\partial \xi_{q}}(\xi)\right]
$$

where $\hat{N}_{i}$ is the shape functions corresponding to the four corner nodes of the master element in $\left(\xi_{1}, \xi_{2}\right)$, and $J^{-1}$ is the inverse of the transpose of the Jacobian matrix. The stiffness matrix is then 
obtained by a numerical integration such as $2 \times 2$ Gaussian rule:

$$
H_{i j}=\int_{\Omega_{e}}\left(\nabla N_{i}\right)^{\mathrm{T}} \mathbf{K} \nabla N_{j} \mathrm{~d} \Omega
$$

\section{GRID ADAPTIVE METHODS}

Although the application of finite element methods to solve shape design problems is standard because of their simplicity and generality, there are few works which thoroughly discuss the quality of finite element approximations, especially the nature of dependency of finite element solutions on the form of the finite element grids. It is certain that convergence and stability of finite element approximations have been established mathematically by, for example, Ciarlet ${ }^{14}$ and Oden and Reddy. ${ }^{15}$ However, quantitative and qualitative analyses of finite element approximate solutions have not been discussed in the past. Recently, grid adaptive finite element methods are introduced in order to grasp both quantitative and qualitative behaviour of the error involved in finite element approximations (see, for example, Shephard ${ }^{16}$ and Babuska et al. ${ }^{17.18}$ ). While there are many adaptive methods to define grids, typical adaptive methods can be classified into three categories: $p$-method, $h$-method, and $r$-method. The first two methods increase the degrees-of-freedom by the increase of the order of polynomials and by the subdivision of elements for grid refinement, respectively. The $r$-method optimally distributes the error into the entire domain by relocating the nodes and thereby changing the shape of elements.

In this section we shall discuss briefly the main aspect of the $r$-and $h$-methods, which serve to eliminate oscillatory behaviour of design boundaries when those adaptations are combined with shape optimization algorithm and used in the iteration process. The minimization of the maximum value of error in $r$ - and $h$-methods is based on two observations:

1. In the finite element model with uniform grid the error measure of approximation is biggest near the singular points and decays rapidly far away from these points.

2. The total amount of strain energy or potential energy is almost the same regardless of the location of nodes inside the domain.

Details can be found in the paper by Diaz et al. ${ }^{19}$

The grid design problem may be defined as follows. Let $E_{e}$ be an error measure of the $e$ th finite element $\Omega_{e}$, and $E$ represents the total number of elements. Then the min-max design problem of grid design for the $r$-method is defined by

$$
\underset{\substack{\text { node } \\ \text { relocation }}}{\operatorname{Min}}\left\{\underset{1 \leqslant e \leqslant E}{\left.\operatorname{Max} E_{e}\right\}}\right.
$$

The min-max problem

$$
\underset{\text { refinement }}{\operatorname{Min}}\left\{\underset{1 \leqslant e \leqslant E}{\operatorname{Max} E_{e}}\right\}
$$

characteristics the $h$-method. For the above problems a necessary condition for the optimality is that the error measure of each finite element must be constant all over the finite element mode, i.e.

$$
E_{e}=\text { constant } \quad e=1, \ldots, E
$$

Note that we are minimizing the maximum error instead of total amount of errors. However, we 
can reduce the total amount of error as well by minimizing the maximum error which bounds the total amount of error $e_{h}$ defined as

$$
e_{h}=\left[\sum_{i=1}^{E} E_{e}^{2}\right]^{1 / 2}
$$

i.e. we have

$$
e_{h} \leqslant E^{1 / 2}\left(\underset{1 \leqslant e \leqslant E}{\operatorname{Max}} E_{e}\right)
$$

where the equality holds when the optimality condition (20) is satisfied.

For the choice of quantitative measure of error in finite element approximations, we shall take the idea that finite element error is bounded by interpolation error. Let us define the error measure $E_{e}$ of seepage flow as

$$
E_{e}=\left[c \int_{-1}^{1} \int_{-1}^{1} \sum K_{i j} \hat{J}_{m i}^{-1} \hat{J}_{n j}^{-1}\left|\nabla_{\xi}^{2} \phi\right|\left|\nabla_{\xi}^{2} \phi\right| J \mathrm{~d} \xi_{1} \mathrm{~d} \xi_{2}\right]^{1 / 2}
$$

(sum in $m, n$ )

in the normalized co-ordinate system $\left(\xi_{1}, \xi_{2}\right)$ for a positive constant $c$. Here, $\hat{J}^{-1}$ is the inverse of the transpose of the Jacobian matrix in the isoparametric relation, $\phi$ is the solution, and $J$ is the Jacobian. Equation (23) is obtained as an upper bound of the interpolation error using

$$
\left[\frac{\partial \phi}{\partial \xi_{j}}-\frac{\partial \phi_{h}}{\partial \xi_{j}}\right]\left(\xi_{1}, \xi_{2}\right) \leqslant c\left|\nabla_{\xi}^{2} \phi\right|, \quad j=1 \text { and } 2
$$

for a positive constant $c \geqslant 0$ independent of $\phi$, where

$$
\nabla_{\xi}^{2}=\frac{\partial^{2}}{\partial \xi_{1}^{2}}+\frac{\partial^{2}}{\partial \xi_{2}^{2}}+2 \frac{\partial^{2}}{\partial \xi_{1} \partial \xi_{2}}
$$

and $\phi_{h}$ is the interpolation of $\phi$. Details of interpolation for finite element approximation error can be found in, for example, Reference 19.

The next step is the approximation of the error measure $E_{e}$ by an error $\hat{E}_{e}$ which can be calculated from the finite element solution $\hat{\phi}_{h}$ because the unknown $\phi$ cannot be used to compute $E_{e}$. To do this the 'second derivatives' of $\hat{\phi}_{h}$ are obtained by taking derivatives of the continuous first derivatives of $\hat{\phi}_{h}$ computed by the least squares method from the discontinuous ones in the four node quadrilateral isoparametric elements. Using the above approximations, an approximated necessary condition (20) to the grid design problem (18) and (19) is defined by

where

$$
\hat{E}_{\mathrm{e}}=\text { constant }, \quad e=1, \ldots, E
$$

$$
\hat{E}_{e}=\left[\int_{-1}^{1} \int_{-1}^{1} \sum K_{i j} \hat{J}_{m i}^{-1} \hat{J}_{n j}^{-1}\left|\nabla_{\xi}^{2} \hat{\phi}_{h}\right|\left|\nabla_{\xi}^{2} \hat{\phi}_{h}\right| J \mathrm{~d} \xi_{1} \mathrm{~d} \xi_{2}\right]^{1 / 2}
$$

(sum in $m, n$ )

Similarly, if the interpolation error on the head, i.e. $\phi-\phi_{h}$, is the basis for the error measure to the grid design problem, $E_{e}$ may be defined by (refer to Reference 20)

$$
E_{e}=\left[\int_{\Omega e} \nabla \phi \cdot \nabla \phi \mathrm{d} \Omega\right]^{1 / 2}
$$


which leads to the approximation

$$
\hat{E}_{e}=\left[\int_{\Omega_{e}} \nabla \hat{\phi}_{h} \cdot \nabla \hat{\phi}_{h} \mathrm{~d} \Omega\right]^{1 / 2}
$$

It must be emphasized that grids are adapted in different ways for different error measures, and that the error measure must be selected carefully to characterize the nature of the given problem.

The major advantage of using the error indicator $E_{e}$ defined in the above is that the existing preprocessor and finite element codes can be used to introduce adaptive schemes of finite element grids. There are many ways to relocate nodes or subdivide elements until the optimality condition ' $\hat{E}_{e}=$ constant' is satisfied. For the $r$-method, one of the relocation schemes is based on

$$
x_{n}=\sum_{e} x_{e}\left(\hat{E}_{e} / A_{e}\right) / \sum_{e}\left(\hat{E}_{e} / A_{e}\right)
$$

where $x_{n}$ is the new location of the $n$th node, $x_{e}$ is the geometric centroid of the element $\Omega_{e}, A_{e}$ is the area of $\Omega_{e}, \hat{E}_{e}$ is the error indicator of $\Omega_{e}$, and the summation is taken over the elements related to the $n$th node. In the $h$-method, elements whose error indicator is larger than some constant times the average error indicator in the domain, are subdivided. Usually, this constant is bigger than 1.0 and its value is set according to the user's choice.

An example is presented to show the differences in the solutions with and without using the grid adaptation. In Figures 2-5 a seepage flow in a foundation with two unsymmetric impermeable walls is considered. Obviously, the velocity of seepage flow becomes infinite at the tips of the walls. Starting from the uniform grid, the adaptive grid are obtained by the $r$ - and $h$-method. In both adaptive grids, elements are accumulated near the tips of the walls. The very large velocities are computed at these tips, while the uniform grid gives a not so large velocity there. However, the

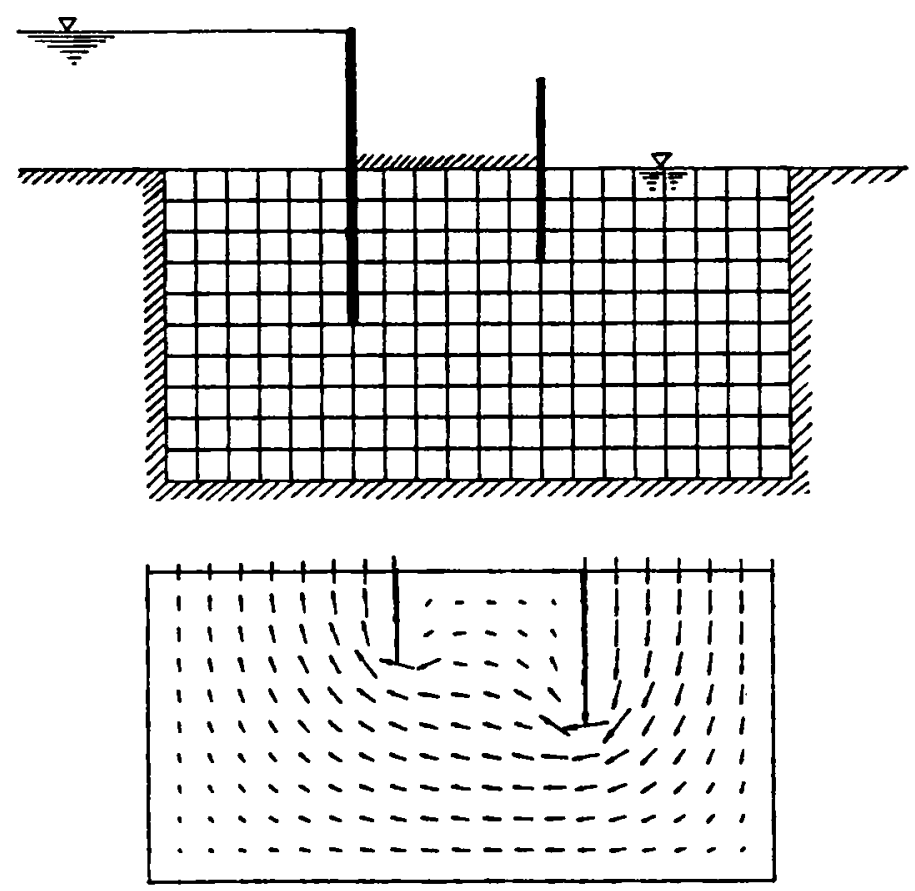

Figure 2. (a) Seepage flow model with a uniform grid; (b) velocity field 
(a)

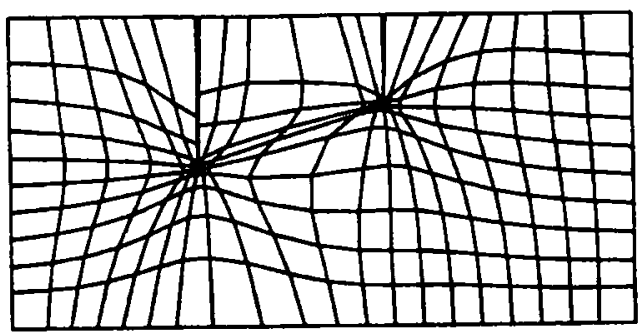

(b)

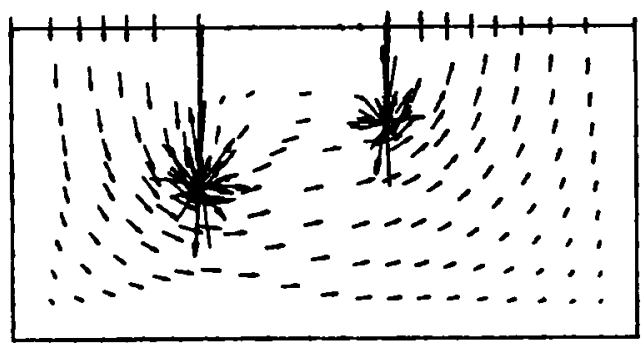

Figure 3. (a) Grid adapted by r-method; (b) velocity field

(a)

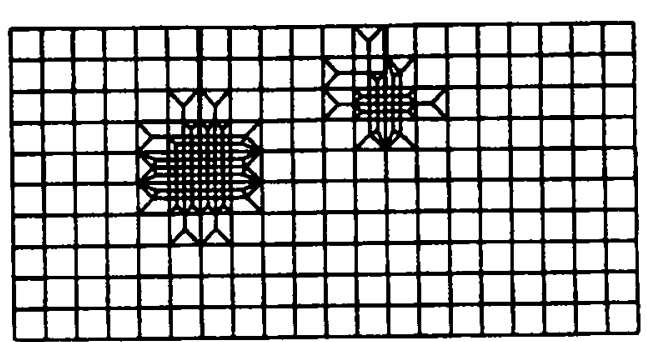

(b)

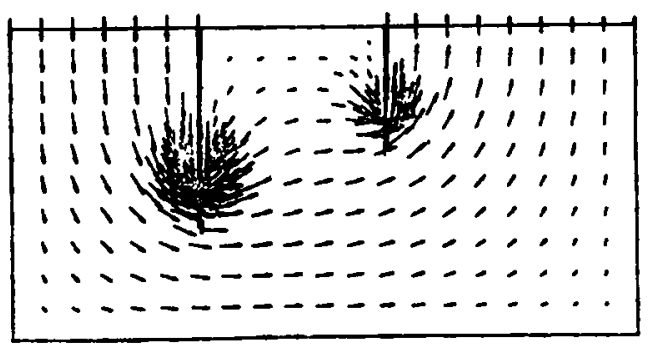

Figure 4. (a) Grid adapted by $h$-method; (b) velocity field

(a)

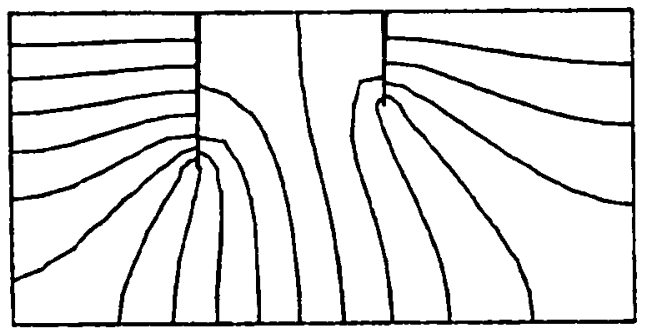

(b)

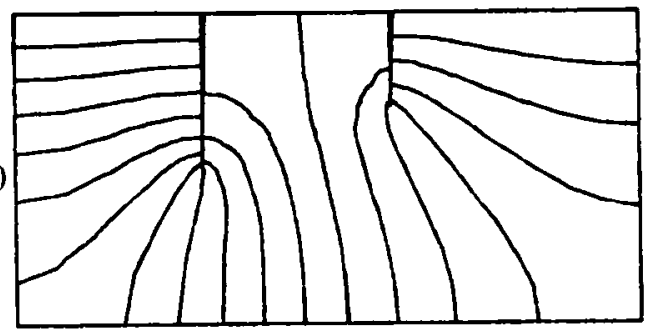

(c)

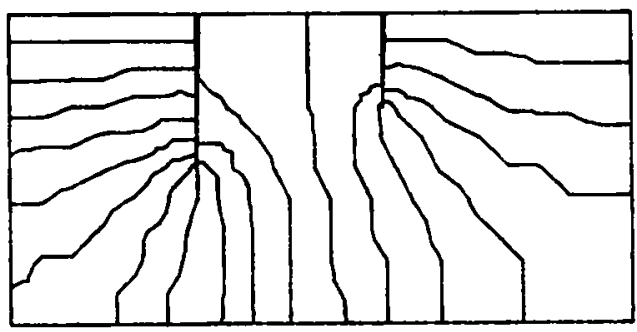

Figure 5. Hydraulic head contours: (a) uniform grid; (b) $r$-adapted grid; (c) $h$-adapted grid 


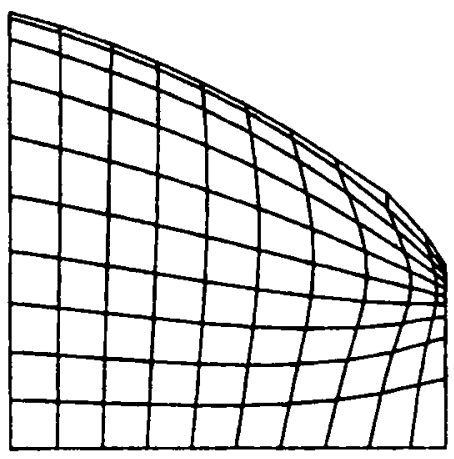

(a)

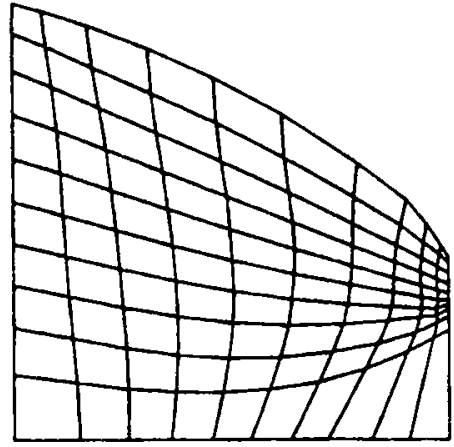

(c)

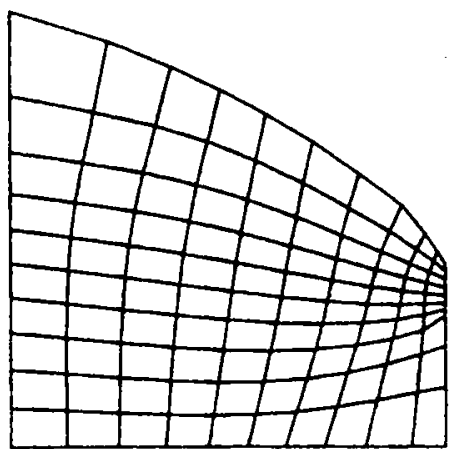

(b)

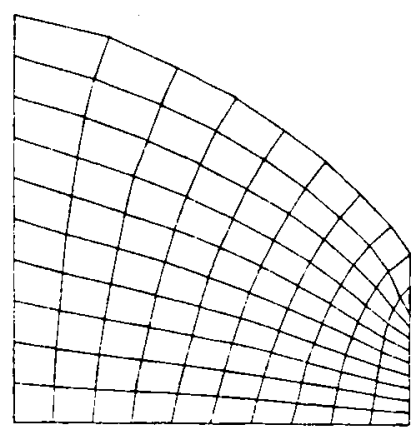

(d)

Figure 6. Adapted grid by $r$-method with error measures: (a) inverse of pressure; (b) derivative of velocity component; (c) Adapted grid by $r$-method with derivative of head as an error measure; (d) by elliptic generator

distribution of hydraulic head contours has not been changed significantly after applying the adaptive methods. These results prove the initial observation, i.e. the total amount of potential energy is almost the same regardless of the grid design.

Figure 6(a-d) demonstrate the dependency of grid adaptation on error measures. In Figure $6(\mathrm{~b})$, the first derivatives of velocity components were used to calculate the error measure, while the inverse of pressure was used in the case of Figure 6(a). The adaptive grid in Figure 6(c) is obtained using the derivatives of the head as an error measure, i.e. equation (29). Since the rectangular dam is homogeneous, the first derivatives of velocity components are equivalent to the second derivatives of the head $\phi$, i.e. the error measure $\hat{E}_{e}$ defined by (27) is applied in Figure 6(b). As shown in Figure 6(d), all the adaptive grids are different from the one obtained by the numerical grid generation method using a system of elliptic differential equation described in Thompson, ${ }^{21}$ although both the head and flow velocity are not affected by the grid in this particular example problem.

Another way to move nodes to have an optimal grid is that introduced by Taylor in $1972 .{ }^{22}$ In this case, using, for example, 8-node elements, mid-nodes are adjusted so that 'singular' behaviour of the solution can be well simulated; see also Bathe and Sussman. ${ }^{23}$

\section{DOMAIN ADAPTIVE METHODS TO SOLVE FREE BOUNDARY PROBLEMS}

Domain adaptive methods have widely been used in many kinds of free boundary problems. In seepage flow problems, Neuman and Witherspoon, ${ }^{3}$ Oden and Kikuchi ${ }^{8}$ and Askew and 
Thatcher ${ }^{24}$ have developed their own methods. In most cases, the idea of domain adaptive methods is the two-step iteration algorithm introduced in Reference 3 for the purpose of satisfying the free boundary conditions, which usually come out to be constant values of some quantities. Consequently, the first step is the calculation of some quantities under the assumption that the free boundaries are fixed, and the second step is the movement of nodes on the free boundaries in the ratio of differences between the calculated quantities and the known constants at the nodes of a finite element model.

In seepage flow problems, two free boundary conditions are given as (6) and (7). Those are: (a) pressure is equal to the atmospheric pressure, in other terms, the hydraulic head at nodes on the free boundary is equal to the vertical height of the nodes; (b) the normal component of velocity is zero along the free boundary. Domain adaptive methods can be used to satisfy the above two conditions or either one of those conditions as long as the final shape of free boundaries is converged so that both conditions are satisfied at the end stage. In finite element methods which solve for the hydraulic head, it is more convenient to manage the first condition than the second one because the accuracy of the hydraulic head is higher than the accuracy of the flow velocity, since the latter is obtained from the derivatives of hydraulic head according to Darcy's law.

In this section, we shall discuss a new but simple method of moving the nodes in the specified direction.

The expression for the iterative scheme to find new co-ordinates of nodes on the free boundaries is

$$
x_{i}^{k+1}=x_{i}^{k}+\Delta x_{i}^{k} \quad i=1, \ldots, N
$$

in $k$ th iteration, where $N$ is the total number of nodes on the free boundary. Here, $\Delta x_{i}^{k}$ is the movement of nodes in the specified direction and this value can be obtained from the movement in the normal direction:

$$
\Delta x_{i}=\frac{\Delta x_{n_{i}}}{\cos \theta_{i}}
$$

in the $k$ th iteration. $\theta_{i}$ is the angle between the unit normal vector $n_{i}$ and the unit direction vector $\alpha$; at the $i$ th node in the specified direction (Figure 7).

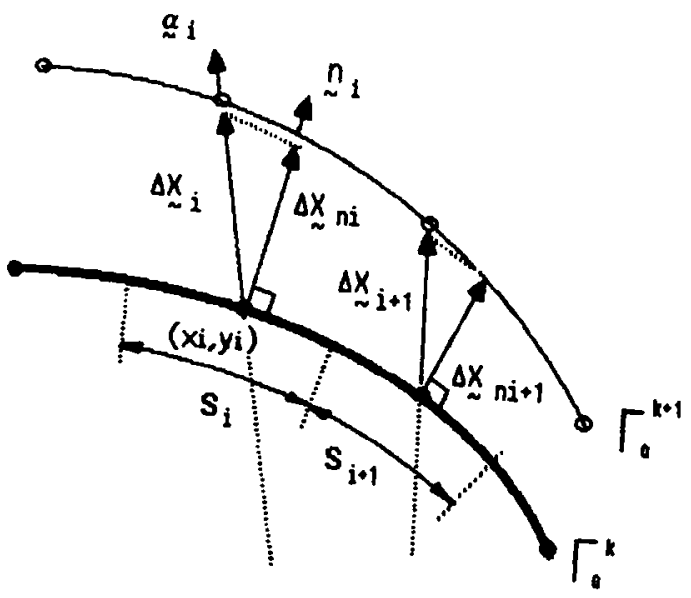

Figure 7. Movement of nodes on the free boundary $\Gamma_{0}^{k}$ at the $k$ th iteration 
For simplicity, iteration superscript $k$ is omitted in the following. The normal movement $x_{n_{i}}$ at the $i$ th node in the $k$ th iteration is obtained from

$$
\begin{aligned}
\Delta x_{n_{i}} & =\frac{\Delta A_{i}}{S_{i}} \\
S_{i} & =\frac{1}{2}\left[l_{i+1}+l_{i}\right]
\end{aligned}
$$

where $\Delta A_{i}$ is the area alloted at the $i$ th node and $l_{i}$ is the length of the $i$ th element on the boundary. The alloted area $\Delta A_{i}$ at the $i$ th node is obtained from the ratio between the hydraulic head $\phi_{i}$ calculated in the previous step of the $k$ th iteration and the $y$-co-ordinate $y_{i}$ :

$$
\Delta A_{i}=\Delta \text { Area }\left[\frac{\frac{\phi_{i}-y_{i}}{y_{i}} \cdot S_{i}}{\sum_{i=1}^{N}\left[\frac{\phi_{i}-y_{i}}{y_{i}}\right] \cdot S_{i}}\right]
$$

$\phi_{i}$ is the value $\phi$ at node $i$, and $\Delta$ Area is the area between the free boundary $\Gamma_{0}^{k+1}$ and $\Gamma_{0}^{k}$. The value of $\Delta$ Area must be large enough at the beginning and diminish as the iteration goes on in order to have convergence. For this purpose we define a percent deviation from the optimum as

$$
\mathrm{DTP}=\left[\frac{1}{N} \sum_{i=1}^{N}\left[S_{i} \cdot \frac{\phi_{i}-y_{i}}{y_{i}}\right]^{2}\right]^{1 / 2} \cdot \frac{1}{S}
$$

using $L^{2}$ norm, where $\bar{S}$ is the average length of elements of the free boundary

$$
\bar{S}=\frac{1}{N-1}\left[\sum_{i=1}^{N} S_{i}\right]
$$

The value for $\Delta$ Area is given by

$$
\triangle \text { Area }=(\text { Total area of domain }) \times C \times \text { DTP }
$$

From our experience, the value of $C$ is between zero and $1 \cdot 0$, although the proper value must be adjusted according to the problem and the speed of convergence. That is, the bigger the $C$ value, the faster the converging speed. However, faster convergence is sometimes accompanied by oscillations of the free boundaries, and possibly by oscillations of the percent deviation as well, as the iterations proceed. On the other hand, a small value of $C$ may result in very slow convergence without oscillations.

An example of the domain adaptive method is presented in Figure $8(a-d)$. A free boundary of a saturated unconfined incompressible two-dimensional flow through a vertical homogeneous dam, is obtained by 10 iterations. With given upstream height 10 and downstream 3 and unit permeability, the calculated flow rate was 4.59 which agrees with exact solution 4.55 from Polubarinova-Kochina ${ }^{25}$ and Liggett's result. ${ }^{26}$ The final results show 0.1 per cent deviation, which means almost zero pressure along the design boundary, and velocity field in Figure 8(c) shows zero normal component on the free boundary and impermeable boundary. The hydraulic head contours in Figure 8(d) are normal to the free boundary and the bottom boundary, which are stream lines. It should be mentioned that the penalty method ${ }^{27}$ was used in the finite element analysis to satisfy the boundary conditions of given values of hydraulic heads on $\Gamma_{0}$, e.g. on the upstream face, the downstream face and the seepage face. 


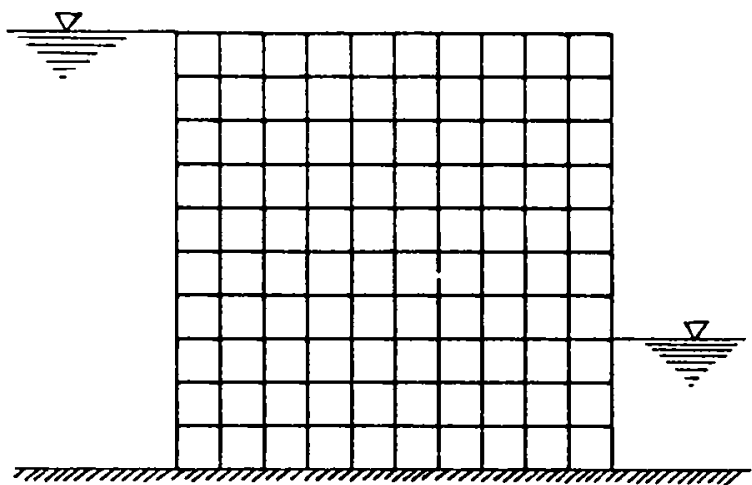

(a)

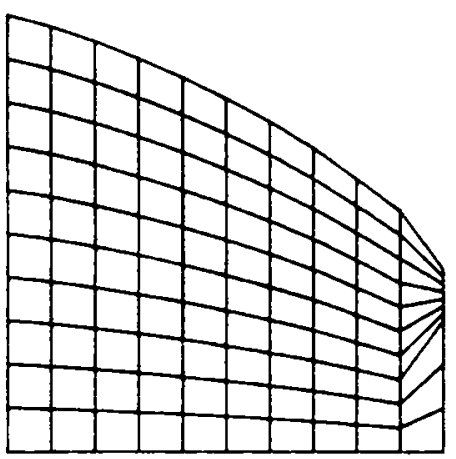

(b)

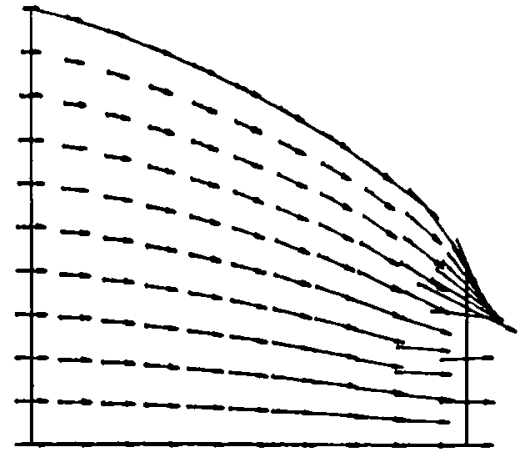

(c)

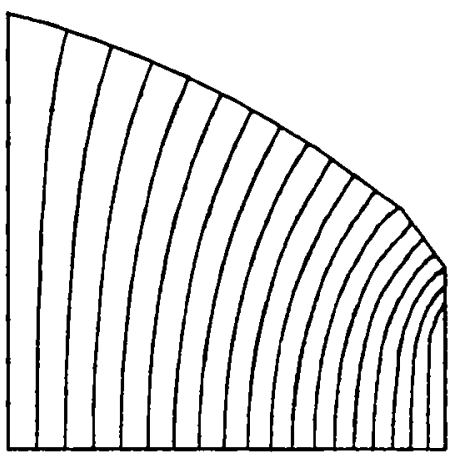

(d)

Figure 8. Shape design of vertical homogeneous dam: (a) initial model; (b) converged shape; (c) velocity field in the converged shape; (d) hydraulic head contours;

\section{COMBINATION OF GRID AND DOMAIN ADAPTIVE METHODS}

As we have discussed, the quality of finite element approximations is strongly affected by finite element grids. In free boundary problems, the accuracy of finite element solutions is very important for the determination of free boundaries by approaching to the final shape iteratively. Finite element grids which are not well designed for singularities of the drastic change of the velocity field frequently lead to boundary shapes with unreasonable oscillation of the boundary shape. In seepage flow problems, oscillations or big jumps of free boundaries usually occur in nonhomogeneous domains of very different permeabilities (see Fig. A.2 in Reference 8).

In this sense, the combination of grid and domain adaptive methods may be necessary not only to avoid oscillations of free boundaries, but also to obtain the best possible computed results. Figure 9 shows an algorithm for the combination of both grid and domain adaptation to solve free boundary problems.

Seepage flow through a non-homogeneous dam is chosen for an example, as shown in Figure 1. Permeabilities are $K_{1}=1, K_{2}=0-05, K_{3}=1$, respectively, and the height of the upstream and of the downstream are $H=16$ and $h=2$. The interface of material 2 and 3 is also taken to be a part of the free stream line. Starting with the uniform grid in Figure 10(a), the shape converged after 10 iterations of the domain adaptive method and contains the oscillation near the interface, see Figure 10(b). Clearly, this shape cannot be the shape of free boundary because of its non-zero 


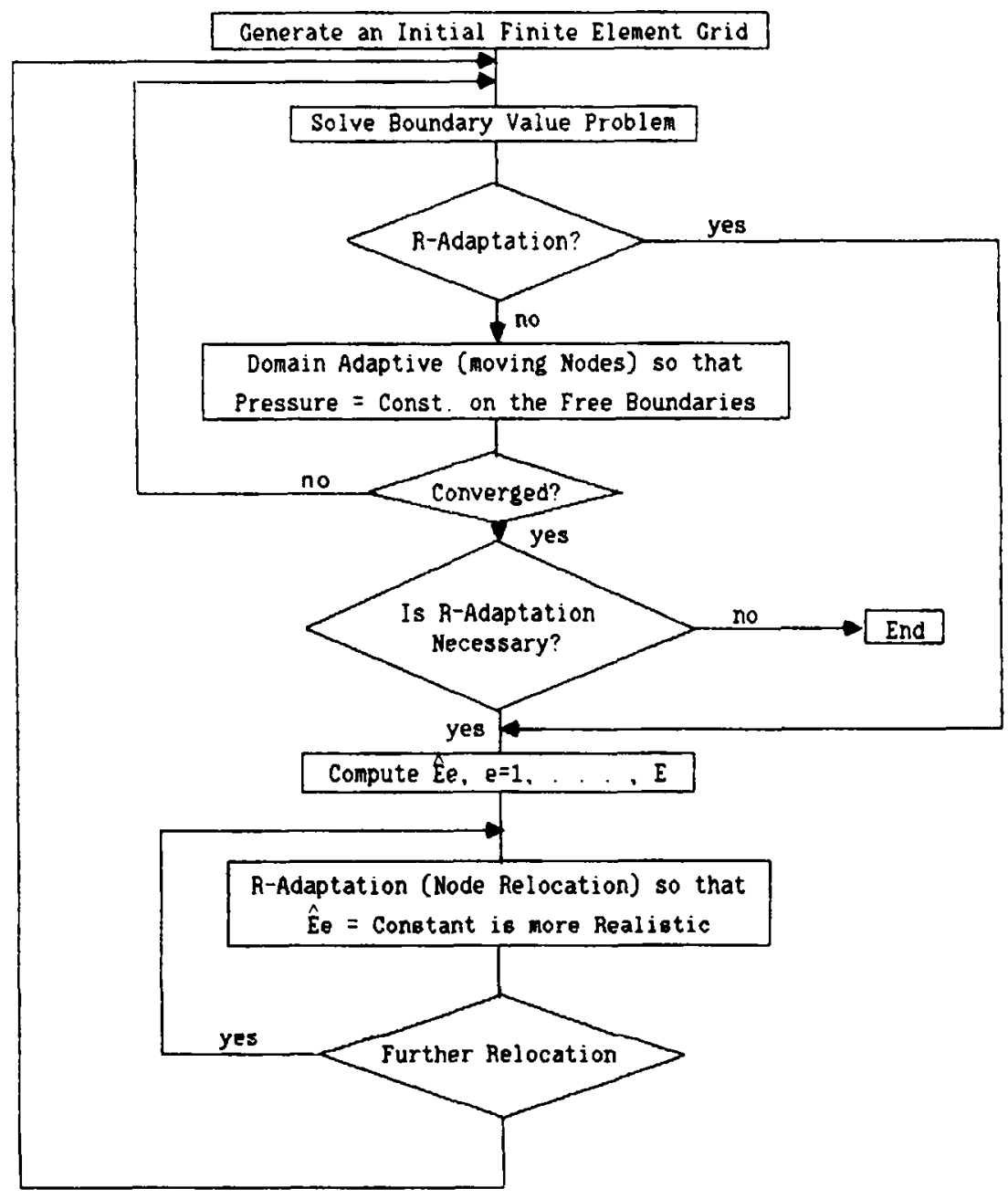

Figure 9. Algorithm for combination of domain and grid adaptive method

normal velocities (Figure 10c), although the pressure reached almost zero on the boundary. However, restarting with the remeshed grid using the $r$-method (Figure 11a), the final shape is obtained after 5 more iterations of domain adaptive scheme and it shows no oscillation (Figure $11 \mathrm{~b}$ ) and the normal velocity is completely vanished on the free boundary (Figure 11c). It is noted that in this example the inverse of absolute value of pressure is used to define the error measure in the grid adaptation.

\section{CONCLUSIONS}

In this paper, we have discussed grid and domain adaptive methods. It has been shown that the combination of grid and domain adaptive methods is useful to solve oscillatory behaviour or unreasonable shape of free boundaries, as well as to provide more consistent velocity fields in the flow through porous media. 


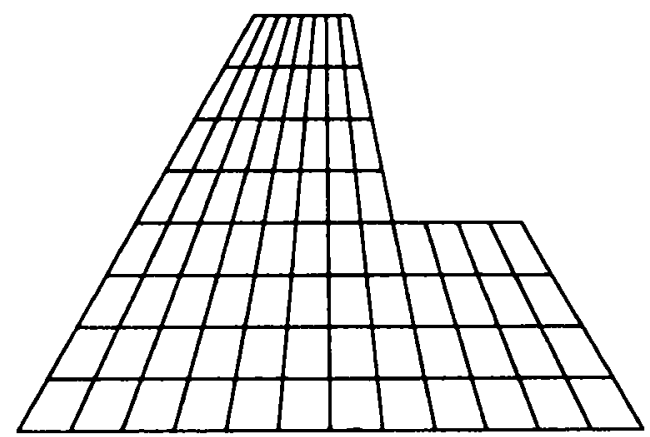

(a)

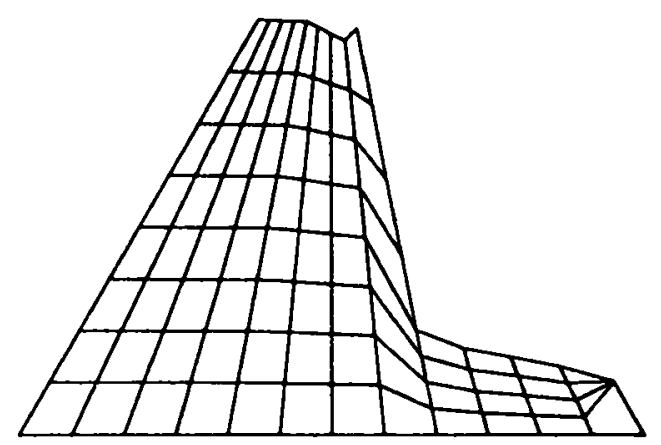

(d)

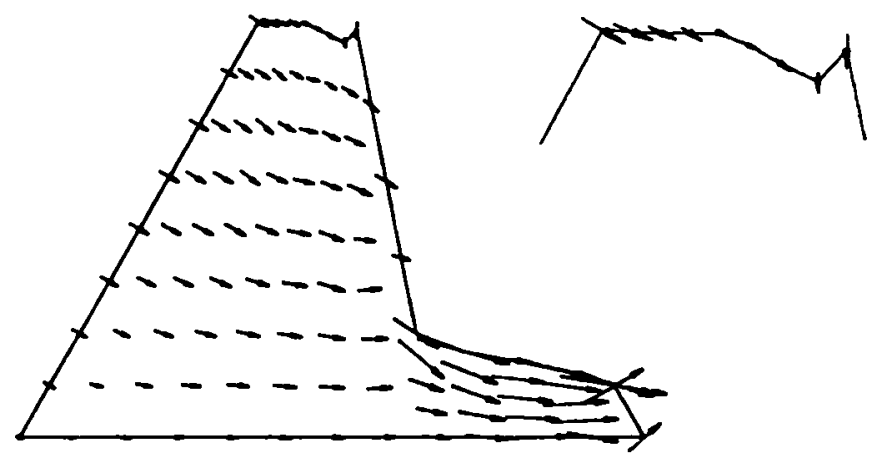

(c)

Figure 10. Non-homogeneous dam with uniform grid: (a) initial model; (b) converged shape before applying $e$-method; (c) velocity field in the shape converged before applying $r$-method

\section{ACKNOWLEDGEMENT}

The authors were supported by NASA Lewis Research Center under Grant NAG 3-388. The authors express their appreciation. 
(a)

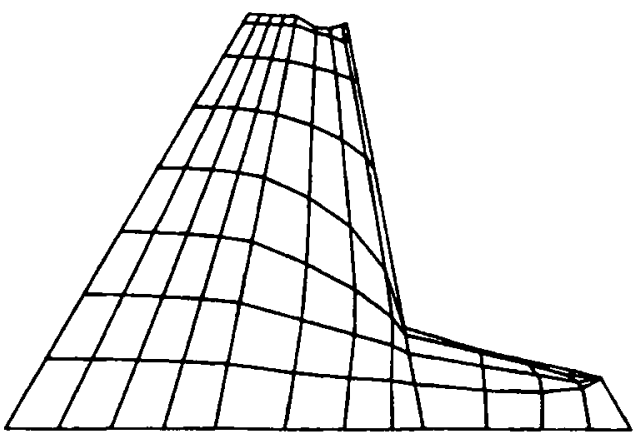

(b)

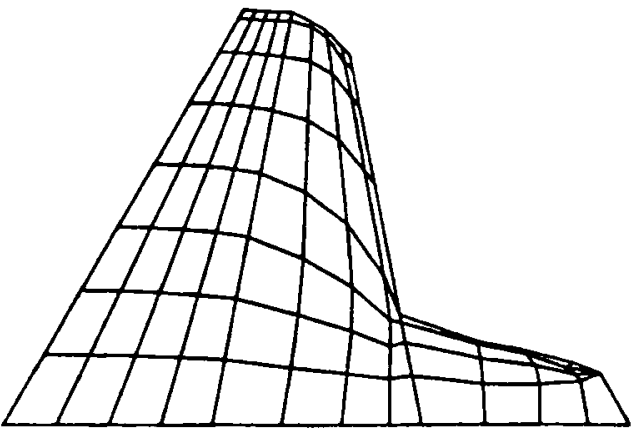

(c)

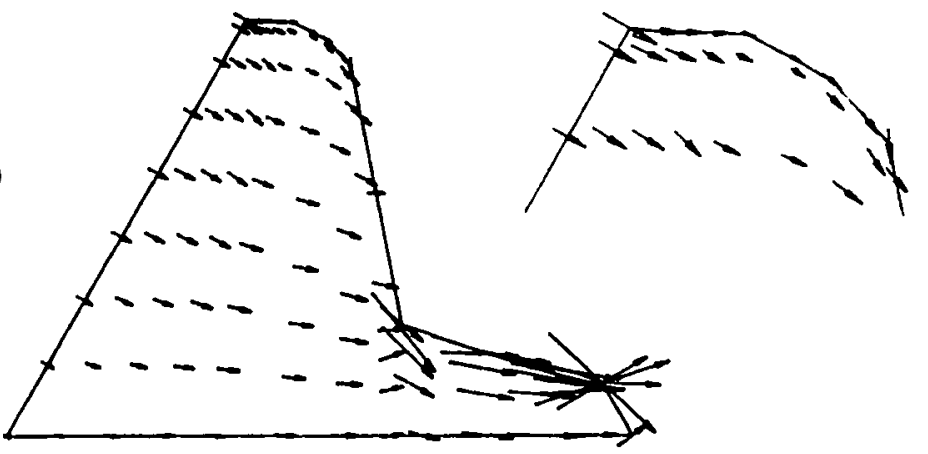

d)

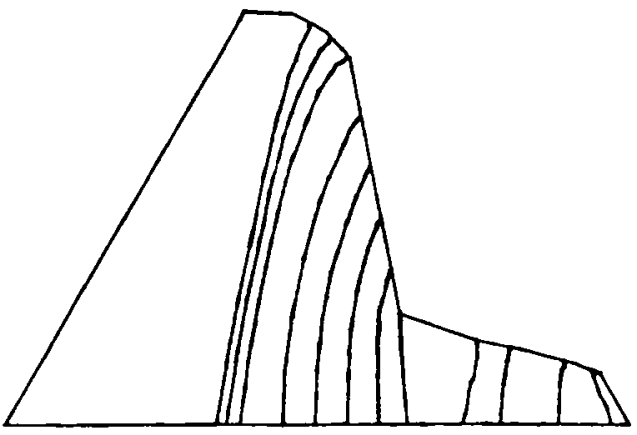

Figure 11. Shape design with remeshed model: (a) restarting shape adapted by $r$-method; (b) final shape converged; (c) velocity field in the final shape; (d) hydraulic head contours in the final shape 


\section{REFERENCES}

1. R. L. Taylor and C. B. Brown, 'Darcy flow solutions with a free surface', J. Hydr. Div., Proc. ASCE, HY2, 25-33 (1967).

2. W. D. L. Finn, 'Finite element analysis of seepage through dams', J. Soil Mech. Div., Proc. ASCE, 41-48 (1967).

3. S. P. Neuman and P. L. Witherspoon, 'Finite element method of analyzing steady seepage with a free surface', $W$ ater Resources Res., 6(3), 889-897 (1970).

4. H. W. Alt, 'A free boundary problem associated with the flow of ground water', Arch. Rat. Mech. Anal., 64, 111 (1977).

5. H. W. Alt, 'The fluid flow through porous media, regularity of the free surface', Manuscripta Math., 21, 255 (1977).

6. H. Brezis, D. Kinderlehrer and G. Stampacchia, Comptes Rendus, 287, ser. A, 711 (1978).

7. P. Le Tallac, Regularization et Resolution Numerique de Problems a Frontiere Libre, IRIA, Paris, 1977.

8. J. T. Oden, and N. Kikuchi, 'Theory of variational inequalities, flow through porous media', Int. J. Eng. Sci., 18 (10), 1173-1284 (1980).

9. C. S. Desai, 'Finite element residual schemes for unconfined flow', Int. j. numer. methods eng., 10, 1415-1418 (1976)

10. C. S. Desai and G. C. Li, 'A residual flow procedure and application for free surface flow in porous media', Adv. Water Res., 6, 27-35 (1972).

11. C. Baiocchi, 'Su un Problema di Frontiera Libera Connesso a Questioni di Idraulica', Annali Math. Pura Appl., 92 (4), 107 (1972).

12. C. Baiocchi, V. Comincioli, E. Magenes and G. A. Pozzi, 'Free boundary problems in the theory of fluid flow through porous media: existence and theorems', Annali Math. Pura Appl., 93 (4), 1-82 (1973).

13. J. C. Bruch, 'A survey of free boundary value problems in the theory of fluid flow through porous media: variational inequality approach, parts I and II', Adv. Water Res., 3, 65-80, 115-124 (1980).

14. P. E. Ciarlet, The Finite Element Method for Elliptic Problems, North-Holland, Amsterdam, 1978.

15. J. T. Oden and J. N. Reddy, An Introduction to the Mathematical Theory of Finite Elements, Wiley-Interscience, New York, 1975.

16. M. S. Shephard, 'Finite element grid optimization,' ASME, Special Publication, PVP-38, ASME, New York (1979).

18. I. Babuska, J. Chandra and J. E. Flaherty (eds), Proc. Symp. on Adaptive Comp. Methods for Partial Differential Equations, SIAM, Philadelphia, 1983.

18. I. Babuska and B. Szabo, 'On the rates of convergence of the finite element methods', Int.j. numer. methods eng., 18, 323341 (1982).

19. A. R. Diaz, N. Kikuch, and J. E. Taylor, 'A method of grid optimization for finite element methods', Comp. Meth. Appl. Mech. Eng., 41, 29-45 (1983).

20. N. Kikuchi and A. R. Diaz, 'Remodelling of finite element grids based on interpolation errors', Int. Conf. on Accuracy Estimates and Adaptive Refinements in Finite Element Computations (ARFEC), Lisbon, Portugal, Vol. 2, 1984, pp. 235-245.

21. J. F. Thompson (ed.) Numerical Grid Generation, Elsevier, Amsterdam, 1983.

22. R. L. Taylor and R. Iding, 'Application of extended variational principles to finite element analysis', in Variational Methods in Engineering, (C. A. Brebbia and H. Tottenham, eds), Vol. I, University of Southampton, pp. 2/54-2/67, 1972.

23. K. J. Bathe and T. Sussman, 'An algorithm for the construction of optimal finite element meshes in linear elasticity', in Computer Methods for Nonlinear Solids and Structural Mechanics, ASME, AMD 54, 1983.

24. S. L. Askew and R. W. Thatcher, 'Calculating the discharge from a porous dam', Comp. Fluids, 12, (1-D) 47-53 (1984).

25. Polubarinova-Kochina, Theory of Ground Water Movement, trans. by J. M. Roger De Wiest, Princeton Univ. Press, N.J., 1962.

26. J. A. Liggett, 'Location of free surface in porous media', J. Hydr. Div., Proc. ASCE, HY4, 353-365 (1977).

27. N. Kikuchi, Finite Element Methods in Mechanics, Cambridge University Press, London, 1986. 